\section{Eficiencia de un apósito innovador en la cura de heridas: reducción de la frecuencia de cambio y del coste semanal por paciente}

\section{The efficiency of an innovative dressing in wound healing: reduction of change frequency and weekly cost per patient}

\author{
Verónica Tiscar González1,* \\ María José Menor Rodríguez² \\ Carlos Rabadán Sainz ${ }^{3}$ \\ Mercedes Fraile Bravo ${ }^{4}$ \\ Grupo Life ${ }^{5}$ \\ Tim Styche ${ }^{6}$ \\ Francisco José Valenzuela Ocaña ${ }^{7}$ \\ Leticia Muñoz García ${ }^{8}$
}
1. Enfermera. Doctorada en Ciencias de la Salud con mención internacional, Universidad de Lleida Supervisora de docencia de Enfermería OSI Araba, Vitoria, España. Grupo de Enfermería clínica y salud comunitaria. Instituto de Investigación Sanitaria (BioAraba).
2. Enfermera. Doctorada en Enfermería. Enfermera educadora en Diabetes en Área Sanitaria de Ourense, Verín y Barco de Valdeorras. Servizo Galego de Saúde (SERGAS). Orense. España.
3. Enfermero. Máster en Investigación en Atención Primaria. Coordinador de Enfermería en Centros de Salud Trinitat y Bilbao del Departamento Valencia-La Fe, Conselleria de Sanitat Universal i Salut Pública, Valencia, España.
4. Enfermera. Doctorada en Enfermería. Coordinadora de cuidados, Servicio Extremeño de Salud (SES), Mérida, España.
5. Miembros del Grupo Life: 5.1. Roberto Abad García. Enfermero. Subdirector de Enfermería, OSI de Bilbao-Basurto, Servicio Vasco de Salud (Osakidetza), Bilbao, España. 5.2. Mónica Arizmendi Pérez. Enfermera en Unidad de Úlceras y Heridas de Atención Primaria, OSI de Bilbao-Basurto, Servicio Vasco de Salud (Osakidetza), Bilbao, España. 5.3. María José Feijoo Janeiro. Enfermera comunitaria en Centro de Salud Xinzo de Limia, Área Sanitaria de Ourense, Verín and Barco de Valdeorras, Servizo Galego de Saúde (SERGAS), Orense, España. 5.4. Ana María Fernández Silvela. Enfermera comunitaria en Centro de Salud de Valle Inclán, Área Sanitaria de Ourense, Verín y Barco de Valdeorras, Servizo Galego de Saúde (SERGAS), Orense, España. 5.5. María Concepción Navarro Penela. Médico, Jefa de programas sociosanitarios de la Dirección General de las personas mayores. Consellería de Igualdad y Políticas Inclusivas, Conselleria de Sanitat Universal i Salut Pública, Valencia, España. 5.6. Mariana Sánchez de Luna Rodríguez. Enfermera en Unidad de Úlceras y Heridas de Atención Primaria, OSI de Bilbao-Basurto, Servicio Vasco de Salud (Osakidetza), Bilbao, España.
6. Healthcare Economics Manager. Smith+Nephew. Hull. Inglaterra.
7. Enfermero. Máster en Acceso y Relaciones con las Administraciones Sanitarias Business Development \& Market Access, Smith+Nephew Advanced Wound Management, Sant Joan despí, Barcelona, España.
8. Enfermera. Doctorada en Enfermería, Clinical Partner Iberia, Smith+Nephew Advanced Wound Management, Sant Joan Despí, Barcelona, España.

${ }^{*}$ Autor para correspondencia.

Correo electrónico: veronica.tiscargonzalez@osakidetza.eus (Verónica Tiscar González).

\section{RESUMEN}

Objetivos: Evaluar el impacto en la frecuencia de cambio de apósito y en el coste del tratamiento de las heridas que tiene la utilización de un apósito de espuma hidrocelular con indicador de cambio y capa enmascaradora de exudado, y valorar la satisfacción del paciente y la opinión del profesional de enfermería con el apósito de estudio frente a los tratamientos previos. Metodología: Estudio observacional, ambispectivo, multicéntrico, realizado en atención primaria y sociosanitaria, en cuatro regiones de España. Participaron pacientes con heridas exudativas, en las que se fuera a sustituir apósitos previos por el apósito del estudio (ALLEVYN Life) según práctica clínica habitual. En cada región, se recogieron y analizaron características de las heridas (antigüedad, localización, tratamientos anteriores, área de la herida), frecuencia de cambio de apósito, coste semanal por paciente (apósitos utilizados y tiempo de enfermería dedicado a curas), satisfacción del paciente y opinión del profesional de enfermería. Resultados: Se incluyó un total de 128 heridas (Galicia: 29; País Vasco: 30; Extremadura: 25,

y Comunidad Valenciana: 44). Tras 4 semanas de uso del apósito del estudio se redujo significativamente la frecuencia de cambio (29-58\%) y el coste semanal por paciente (apósitos utilizados: 11-61\%; apósitos +

Recibido el 6 de julio de 2020; aceptado el 8 de octubre de 2020.

Objectives: To assess the impact on the dressing change frequency and cost of wound management of using a hydrocellular foam dressing with change indicator and exudate masking layer, and to evaluate the patient's satisfaction and nurse's opinion of the study dressing versus previous treatments. Methods: Observational, ambispective, multicenter study conducted in primary care and social health care, in four regions of Spain. Patients with acute or chronic exuding wounds, in which previous dressings were to be replaced by the study dressing (ALLEVYN Life) according to usual clinical practice, participated. In each region, wound characteristics (evolution period, location, previous dressings, wound area), dressing change frequency, weekly cost per patient (dressings used and nursing time spent), patient satisfaction and nursing staff opinion were collected and analysed. Results: A total of 128 wounds were included (Galicia: 29; Basque Country: 30; Extremadura: 25; and Valencia: 44). After four weeks of use of the study dressing the frequency of change (29-58\%) and the weekly cost per patient were significantly reduced (dressings 
tiempo de enfermería: 27-59\%). En la mayoría de regiones, la reducción del área fue superior al 30\%. La experiencia global de los pacientes mejoró con el apósito de estudio frente al tratamiento previo, y fue buena o muy buena en el 60-78\% de los casos. En todas las regiones, el profesional de enfermería consideró los apósitos ALLEVYN Life superiores en rendimiento global, indicación de idoneidad de cambio y enmascaramiento del exudado. Conclusiones: El uso del apósito con indicador de cambio y capa enmascaradora disminuyó la frecuencia de cambio de apósito, lo que permitió prolongar los intervalos entre visitas y reducir costes asociados. Al mismo tiempo, mejoró la opinión del profesional de enfermería, y aumentó la satisfacción del paciente.

PALABRAS CLAVE: Eficiencia, apósitos, coste, lesión por presión, úlcera de pierna, cicatrización, enfermería, atención primaria y atención sociosanitaria. used: 11-61\%; dressings + nursing time: $27-59 \%)$. In most regions, the area reduction was more than $30 \%$. The overall patient satisfaction with the study dressing was improved, being good or very good in $60-78 \%$ of cases. In all regions, nurses considered ALLEVYN Life dressings superior in overall performance, indication of change adequacy and exudate masking. Conclusions: The use of the dressing with change indicator and masking layer decreased the frequency of dressing changes, allowing for longer intervals between visits and reducing associated costs. At the same time, they improve the opinion of the nursing staff, and increased patient satisfaction.

KEYWORDS: Efficiency, dressings, cost, pressure injure, leg ulcer, wound healing, nurse, primary care, geriatric care.

\section{- INTRODUCCIÓN}

Actualmente, las heridas suponen un importante problema de salud de elevado impacto para el sistema sanitario, tanto desde el punto de vista de la salud poblacional, ya que afecta a la calidad de vida de las personas que las padecen, como del coste económico total invertido en su manejo. Las políticas sanitarias deberían enfocarse principalmente en investigación, prevención y tratamiento de las heridas ${ }^{1}$.

En algunos países europeos se estima que entre 3 y 4 personas de cada 1000 habitantes sufren heridas ${ }^{1}$, que pueden derivar en heridas complejas. Mientras las heridas agudas ocurren de forma repentina y su cicatrización suele ser óptima, como es el caso de quemaduras o heridas quirúrgicas, las heridas complejas tienen una tendencia a la cronificación; las más frecuentes son las lesiones por presión (LPP) y las úlceras en las extremidades inferiores (EE. II.)2.

En España, la prevalencia de las LPP en atención sociosanitaria y atención primaria (AP) se encuentra en torno al 4,0\% y $4,8 \%{ }^{3}$, respectivamente. La prevalencia de úlceras en EE. II. en centros sociosanitarios se acerca al 3,6\% de los ingresados ${ }^{4}$ y en AP, en torno al $0,1-0,3 \%$ de la población general ${ }^{4,5}$. Dichas heridas presentan mayor prevalencia en pacientes mayores de 65 ańos $^{4}$.

El tratamiento de las heridas requiere un elevado uso de recursos sanitarios, y comprende aproximadamente el 5,2\% del total del gasto sanitario nacional ${ }^{6}$.

Se estima que el uso de apósitos y materiales representa cerca de un $15 \%$ del total de los recursos sanitarios asociados al manejo de las heridas, mientras el tiempo de enfermería supone un 30-35\%, y las hospitalizaciones, aproximadamente un 50\% ${ }^{1}$.

La AP atiende el mayor porcentaje de pacientes con heridas complejas ${ }^{3}$, donde el uso de recursos recae principalmente en los apósitos utilizados y en el tiempo de enfermería dedicado al cambio de apósito, y a actividades como la limpieza, el desbridamiento y la evaluación de las heridas ${ }^{1,7}$. Además, este tiempo de enfermería se ve aumentado cuando se realizan visitas domiciliarias, donde influye, entre otros factores, la dispersión geográfica de la población de referencia.

La frecuencia de cambio de apósito se considera uno de los principales generadores de costes en el manejo de heridas, junto con el tiempo de cicatrización y la incidencia de complicaciones ${ }^{1}$. Una inadecuada frecuencia de cambio podría retrasar la cicatrización, incrementar el exudado y favorecer las infecciones ${ }^{8-10}$. Por otra parte, implica mayor número de visitas y materiales utilizados ${ }^{8}$. Esto supone un impacto significativo sobre el bienestar y la calidad de vida del paciente y conlleva una elevada carga a nivel asistencial ${ }^{1,11}$.

El apósito utilizado debe tener unas características que se adecuen al paciente y la herida, con el objetivo de favorecer la cicatrización ${ }^{1,12}$. Por otra parte, con el desarrollo del paradigma de cura en ambiente húmedo (CAH), el apósito debe tener capacidad de manejar el exudado, de forma que favorezca el ambiente húmedo y permita un intervalo adecuado entre los cambios, generando de esta forma un mayor confort en los cuidados ${ }^{13}$.

El sistema sanitario se enfrenta al reto de ofrecer una atención sanitaria de calidad para el control de las heridas, al mismo tiempo que mejora su eficiencia, optimizando los recursos sanitarios asociados. Recientemente, el desafío para la atención sanitaria que ha supuesto la pandemia de COVID-19 refleja la necesidad de adecuar los intervalos entre visitas disminuyendo la frecuencia de cambios, y la importancia de que el profesional sanitario empodere al paciente o cuidador en el autocuidado de las heridas ${ }^{14-16}$.

Una estrategia para alcanzar estos objetivos podría ser la de disponer en la práctica clínica de apósitos innovadores que permitan no solo alcanzar los resultados clínicos esperados, sino reducir el número de cambios innecesarios para: mejorar la eficiencia en la práctica clínica habitual y minimizar los riesgos en situaciones que así lo requieran.

Actualmente, se dispone de un apósito de espuma hidrocelular que promueve la cicatrización y facilita la retención del exudado mediante un núcleo altamente absorbente. El apósito incorpora un sistema innovador con indicador de cambio que informa de la necesidad de sustituir el apósito. Dispone de una barrera semipermeable que bloquea líquidos y bacterias para evitar la infección. Su núcleo absorbente enmascara el exudado, reduce el impacto social que supone la herida y mejora la satisfacción del paciente. Su efectividad y seguridad han sido estudiadas previamente en paciente con LPP ${ }^{17,18}$.

\section{- OBJETIVOS}

Objetivo primario: evaluar la eficiencia en práctica clínica real en el tratamiento de heridas, de un apósito de espuma hidrocelular con indicador de cambio y capa enmascaradora de exudado, en términos de frecuencia de cambio de apósito y coste semanal por paciente. 
Objetivo secundario: valorar la calidad asistencial en práctica clínica real en el tratamiento de heridas, en términos de satisfacción del paciente y opinión de los profesionales de enfermería.

Objetivo exploratorio: analizar el cambio en el área de la herida tras el seguimiento.

\section{- MATERIAL Y MÉTODOS}

\section{Diseño del estudio}

Estudio observacional, ambispectivo, multicéntrico, en vida real, llevado a cabo entre noviembre de 2017 y marzo de 2019 en centros de salud y residencias sociosanitarias de: Galicia (2 centros de salud), País Vasco (8 centros de salud), Extremadura (8 centros de salud) y Comunidad Valenciana (6 centros de salud y 6 residencias sociosanitarias).

\section{Unidad de estudio}

Población: pacientes con heridas que reciben curas por parte de las enfermeras de los centros participantes.

Criterios de selección: se incluyeron todos los pacientes que cumplieron los siguientes criterios de inclusión: pacientes de 18 años o más; con heridas poco profundas por segunda intención en fase de granulación, heridas crónicas o agudas exudativas, de espesor total o parcial; que reciben curas con apósito de espuma o cura en ambiente húmedo; con expectativa de seguimiento de 3 semanas o más, y consentimiento informado firmado.

Se excluyeron: todos los pacientes con alguna contraindicación del apósito aplicable; pacientes que participaban en ensayos clínicos durante el estudio o en los 6 meses previos, y con úlceras cuya localización y/o etiología precisaban cambios de apósito por motivos no relacionados con las características de este, tales como heridas en sacro.

Tamaño de la muestra: en cada región, se llevó a cabo un estudio piloto con aproximadamente 30 pacientes. El tamaño de la muestra se estableció basándose en estudios previos similares ${ }^{7,19}$ y en las recomendaciones de la literatura científica para el cálculo de la muestra en estudios piloto, en los cuales generalmente se recomendó una muestra de 30 sujetos ${ }^{20,21}$.

\section{Procedimientos del estudio}

Durante el estudio se realizaron dos visitas (fig. 1). Previamente se informó a los pacientes del estudio y se recogió el consentimiento informado. En la visita inicial se evaluaron las heridas, se recogieron variables relacionadas con los apósitos utilizados en la semana previa, así como la satisfacción del paciente con dicho tratamiento. Durante la visita se sustituyeron los apósitos previos por el apósito del estudio ALLEVYN Life (Smith \& Nephew, Hull, UK), que podía aplicarse tanto como apósito primario como secundario, según la práctica clínica habitual. La decisión de cambiar de apósito se basó en la última semana de tratamiento y dependió exclusivamente de criterios clínicos. El tamaño del apósito

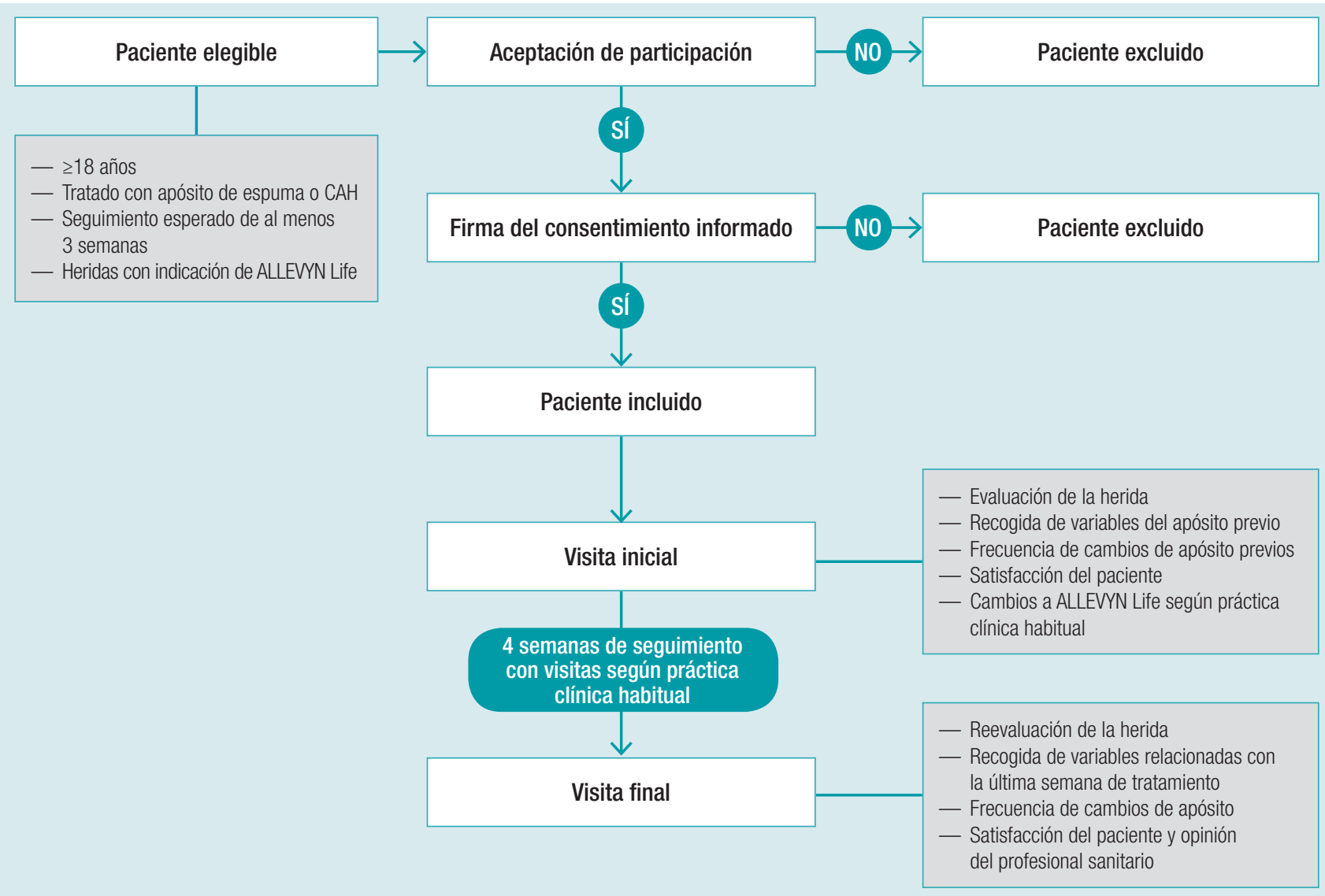

Figura 1. Diagrama de flujo del estudio.

CAH: cura en ambiente húmedo. 
del estudio varió en función del tamaño de la herida $(10,3 \times 10,3 \mathrm{~cm}$, $12,9 \times 12,9 \mathrm{~cm}, 15,4 \times 15,4 \mathrm{~cm}$ o $21 \times 21 \mathrm{~cm}$ ).

Tras la sustitución del apósito, los pacientes acudieron a las visitas programadas para la evaluación y las curas de las lesiones, si era preciso según indicador de cambio o necesidad relacionada con la lesión, durante 4 semanas, siguiendo la práctica clínica habitual. Cada vez que fue necesario cambiar el apósito, este se aplicó según las instrucciones del fabricante. En la semana 4 se realizó una segunda y última visita para reevaluar las heridas y recoger las variables relativas a la última semana de tratamiento, junto con la opinión del profesional y la satisfacción del paciente.

\section{Recogida de datos y variables del estudio}

Fue realizada a través de un cuaderno de recogida de datos electrónico a partir de datos de la historia clínica del paciente y de cuestionarios ad hoc. Si los pacientes recibían atención para múltiples heridas, cada herida era informada y analizada individualmente.

La variable principal fue la frecuencia de cambio de apósito, definida como el número de cambios realizados por semana, medidos durante la semana previa a la inclusión en el estudio y en la última semana de seguimiento (semana 4).

En la visita inicial se registraron:

- Tipo de centro sanitario (centro de salud/sociosanitario).

- Características de las heridas: tipo y área de la herida $\left(\mathrm{en}^{\mathrm{cm}}{ }^{2}\right)$, tiempo de evolución y presencia de infección.

- Variables relacionadas con los apósitos utilizados durante la semana previa: tipo, tamańo y número de apósitos utilizados en cada cambio, número de cambios realizados y localización (primarios/secundarios).

- Satisfacción del paciente con la experiencia global del tratamiento de su herida, mediante un cuestionario ad hoc con una escala de 5 puntos de tipo Likert (muy mala/mala/adecuada/buena/muy buena).

En la visita final, se registraron:

- Área de la herida $\left(\mathrm{en} \mathrm{cm}^{2}\right)$.

- Variables relacionadas con el apósito del estudio: tamaño y número de apósitos utilizados en cada cambio, número de cambios realizados, localización (primarios/secundarios), tipo y número de apósitos utilizados en combinación.

- Satisfacción del paciente (misma escala que en la visita inicial) y probabilidad de recomendar el servicio del cuidado de las heridas a amigos y familiares (muy improbable/improbable/probable/muy probable).

- Opinión del profesional de enfermería sobre ALLEVYN Life en comparación con apósitos previos, mediante un cuestionario ad hoc con escala de tipo Likert de 3 puntos (mejor/igual/peor). Se valoró: capacidad de involucrar al paciente/cuidador en las curas, rendimiento global, facilidad en la retirada, indicación de idoneidad de cambio, enmascaramiento del exudado, capacidad de adaptación a la herida y facilidad de uso. Adicionalmente, indicaron si recomendarían el uso del apósito en su organización.

Para describir el impacto económico del cambio de apósito, se calculó el coste semanal medio asociado al uso de los apósitos previos y al del apósito del estudio. El análisis del coste semanal por paciente se realizó desde la perspectiva del Sistema Nacional de Salud, excluyendo heridas atendidas en centros sociosanitarios. Para el cálculo, se consideró el coste de los apósitos utilizados y el tiempo de enfermería dedicado a las curas.
Con el objetivo de estimar el coste semanal por paciente en función del material utilizado, se multiplicaron los precios unitarios de los apósitos por el número de apósitos utilizados y el número de cambios por semana. Los precios unitarios de los apósitos utilizados antes y durante el estudio fueron proporcionados por los equipos investigadores de cada región.

Para la estimación en función del coste de la visita de enfermería para curas ambulatorias, se obtuvieron los costes unitarios en cada región (Galicia: 42,13 €; País Vasco: 24,00 €, y Extremadura: 12,89 €) y se multiplicaron por la frecuencia de $\operatorname{cambios}^{22-25}$. En la Comunidad Valenciana no se tuvo en cuenta este tipo de coste debido a las diferencias en la gestión del coste de curas de enfermería entre centros de salud y sociosanitarios.

\section{Análisis estadístico}

Se realizó un análisis descriptivo de los datos recogidos para cada región. Para describir variables cualitativas, se calcularon frecuencias relativas y absolutas, mientras que, para variables cuantitativas, se calcularon medidas de centralidad y dispersión (media y desviación estándar [DE]).

Se comparó la frecuencia de los cambios de apósito, el área de la herida y el coste semanal por paciente antes y después del cambio de apósito. Dicha comparación se realizó mediante la prueba T y la de Wilcoxon, en función de la normalidad. Se consideró estadísticamente significativo un valor de $p<0,05$.

Se analizaron los datos de todas las heridas incluidas en el estudio que completaron el seguimiento de las 4 semanas, mediante el software SAS (versión 9.4).

\section{Aspectos éticos}

El estudio fue aprobado por los siguientes comités éticos de investigación: CEIm del Hospital Clínico Universitario de Valencia; Comité Autonómico de Evaluación de Estudios Postcomercialización Observacionales, de Seguimiento Prospectivo con productos sanitarios con marcado CE (CAEPRO); CEIC de Badajoz; Comité Autonómico de Ética de la Investigación de Galicia, y CEIC del Hospital Universitario de Basurto. Se realizó según las guías de buena práctica clínica y en cumplimiento de los principios de la Declaración de Helsinki.

\section{RESULTADOS}

\section{Estudio piloto en el Área Sanitaria de Ourense-Verín-Barco de Valdeorras (Galicia)}

\section{Características DE LAS HERIDAS}

Se incluyeron 17 pacientes con 29 heridas de diferente tipología (tabla 1) y una evolución de 6 meses o menos en el $65,5 \%(n=19)$.

\section{IMPACTO EN LA PRÁCTICA CLÍNICA}

El apósito del estudio se utilizó como apósito secundario en el 67,9\% $(\mathrm{n}=19)$ de las heridas, y el desbridamiento y la sospecha de infección fueron los motivos principales para esta indicación. En estos casos se utilizaron primariamente fibras gelificantes y cremas barrera.

Frecuencia de cambio de apósito. Con el uso del apósito del estudio, se obtuvo una reducción estadísticamente significativa en la frecuencia de cambio semanal de un 29\% (tabla 2). 
Tabla 1. Características de las heridas evaluadas en cada estudio piloto

\begin{tabular}{|c|c|c|c|c|c|}
\hline \multirow{2}{*}{\multicolumn{2}{|c|}{ Características }} & Galicia & País Vasco & Extremadura & C. Valenciana \\
\hline & & $\%(\mathrm{~N}=29)$ & $\%(\mathrm{~N}=30)$ & $\%(\mathrm{~N}=25)$ & $\%(\mathrm{~N}=44)$ \\
\hline \multirow{10}{*}{ Tipología } & Úlceras en EE.II. & $48,3 \%(14 / 29)$ & $50,0 \%(15 / 30)$ & $40,0 \%(10 / 25)$ & $27,3 \%(12 / 44)$ \\
\hline & Traumáticas & $17,2 \%(5 / 29)$ & $10,0 \%(3 / 30)$ & - & - \\
\hline & LPP & $13,8 \%(4 / 29)$ & $13,3 \%(4 / 30)$ & $40,0 \%(10 / 25)$ & $50,0 \%(22 / 44)$ \\
\hline & Quemaduras/abrasiones & $6,9 \%(2 / 29)$ & - & - & $4,5 \%(2 / 44)$ \\
\hline & Cortes/laceraciones & $6,9 \%(2 / 29)$ & - & - & - \\
\hline & Pie diabético & - & $13,3 \%(4 / 30)$ & $4,0 \%(1 / 25)$ & $2,3 \%(1 / 44)$ \\
\hline & Quirúrgicas & - & - & $12,0 \%(3 / 25)$ & $4,5 \%(2 / 44)$ \\
\hline & Hematoma & - & - & $4,0 \%(1 / 25)$ & 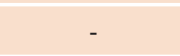 \\
\hline & Desgarro cutáneo & & & - & $6,8 \%(3 / 44)$ \\
\hline & Otras & $6,9 \%(2 / 29)$ & $13,3 \%(4 / 30)$ & - & $4,5 \%(2 / 44)$ \\
\hline \multirow{3}{*}{ Área de la herida } & $<2 \mathrm{~cm}^{2}$ & $35,7 \%(10 / 28)$ & $44,8 \%(13 / 29)$ & $4,0 \%(1 / 25)$ & $11,4 \%(5 / 44)$ \\
\hline & $2-10 \mathrm{~cm}^{2}$ & $60,7 \%(17 / 28)$ & $41,4 \%(12 / 29)$ & $52,0 \%(13 / 25)$ & $59,1 \%(26 / 44)$ \\
\hline & $>10 \mathrm{~cm}^{2}$ & $3,6 \%(1 / 28)$ & $13,8 \%(4 / 29)$ & $44,0 \%(11 / 25)$ & $29,5 \%(13 / 44)$ \\
\hline \multirow{2}{*}{$\begin{array}{l}\text { Presencia de } \\
\text { infección }\end{array}$} & Sí & $44,8 \%(13 / 29)$ & $26,7 \%(8 / 30)$ & $29,2 \%(7 / 24)$ & $22,0 \%(9 / 41)$ \\
\hline & No & $55,2 \%(16 / 29)$ & $73,3 \%(22 / 30)$ & $70,8 \%(17 / 24)$ & $78,0 \%(32 / 41)$ \\
\hline
\end{tabular}

EE.Il:: úlceras en la extremidad inferior; LPP: lesiones por presión.

Tabla 2. Impacto del apósito ALLEVYN Life en la frecuencia de cambio, el área de la herida y el coste semanal por paciente (Galicia)

\begin{tabular}{|l|c|c|c|c|}
\hline & Visita inicial (media \pm DE) & Visita final (media \pm DE) & Reducción & Valor de $p$ \\
\hline Número de cambios de apósito & $2,1 \pm 0,4$ & $1,5 \pm 0,5$ & $29,0 \%$ & $<0,0001$ \\
\hline Área de la herida $\left(\mathrm{cm}^{2}\right)$ & $4,4 \pm 5,0$ & $2,7 \pm 5,1$ & $38,6 \%$ & 0,0109 \\
\hline Coste apósito semanal por paciente $(€)$ & $9,1 \pm 3,8$ & $8,2 \pm 5,1$ & $10,7 \%$ & 0,1812 \\
\hline Coste apósito-enfermería semanal por paciente $(€)$ & $99,2 \pm 15,3$ & $72,1 \pm 26,1$ & $27,4 \%$ & 0,0097 \\
\hline
\end{tabular}

Satisfacción del paciente. Los pacientes valoraron su experiencia global con el tratamiento previo de sus heridas como buena-muy buena en un 20,8\% de los casos, mientras que, tras el uso del apósito del estudio, fue buena-muy buena en el 60,7\%. Además, en el $82 \%$ de los casos, el paciente indicó que sería "probable" o "muy probable" su recomendación del servicio a amigos o familiares en caso de necesitarlo.

Opinión del profesional de enfermería. De acuerdo con la opinión de las enfermeras, en términos de "rendimiento global", "indicación de idoneidad de cambio" y "enmascaramiento del exudado", el apósito del estudio fue superior a los utilizados previamente (fig. 2).

En el 79,3\% ( $n=23)$ de los casos, la enfermera recomendaría el uso de ALLEVYN Life en su organización.

IMPACTO EN LOS RESULTADOS CLÍNICOS

Con el apósito del estudio, se obtuvo una reducción estadísticamente significativa del área de la herida del $38,6 \%$ a las 4 semanas (tabla 2).

\section{IMPACTO ECONÓMICO}

Considerando únicamente el material utilizado en las curas, el coste semanal por paciente se redujo un $10,7 \%$ con el uso de ALLEVYN Life, aunque la reducción no fue estadísticamente significativa. Sin embargo, teniendo en cuenta adicionalmente el coste del tiempo de enfermería, con el apósito del estudio, se obtuvo una reducción estadísticamente significativa del $27,4 \%$ en el coste semanal (tabla 2).

\section{Estudio piloto en la OSI Bilbao-Basurto (Osakidetza)}

\section{CARACTERÍSTICAS DE LAS HERIDAS}

Se incluyeron 23 pacientes con 30 heridas de diferente tipología (tabla 1). La mitad de las heridas presentaron una evolución superior a 6 meses.

IMPACTO EN LA PRÁCTICA CLÍNICA

El apósito del estudio se utilizó como apósito secundario en la mayoría de las heridas $(86,2 \% ; n=25)$, siendo los moduladores de proteasa, el yodo y los antimicrobianos, los productos más utilizados de forma primaria.

Frecuencia de cambio de apósito. Con el uso del apósito del estudio, se obtuvo una reducción estadísticamente significativa en la frecuencia de cambio semanal del 43,5\% (tabla 3). 


\section{En comparación con el tratamiento anterior, ¿cómo calificaría los apósitos ALLEVYN Life?}

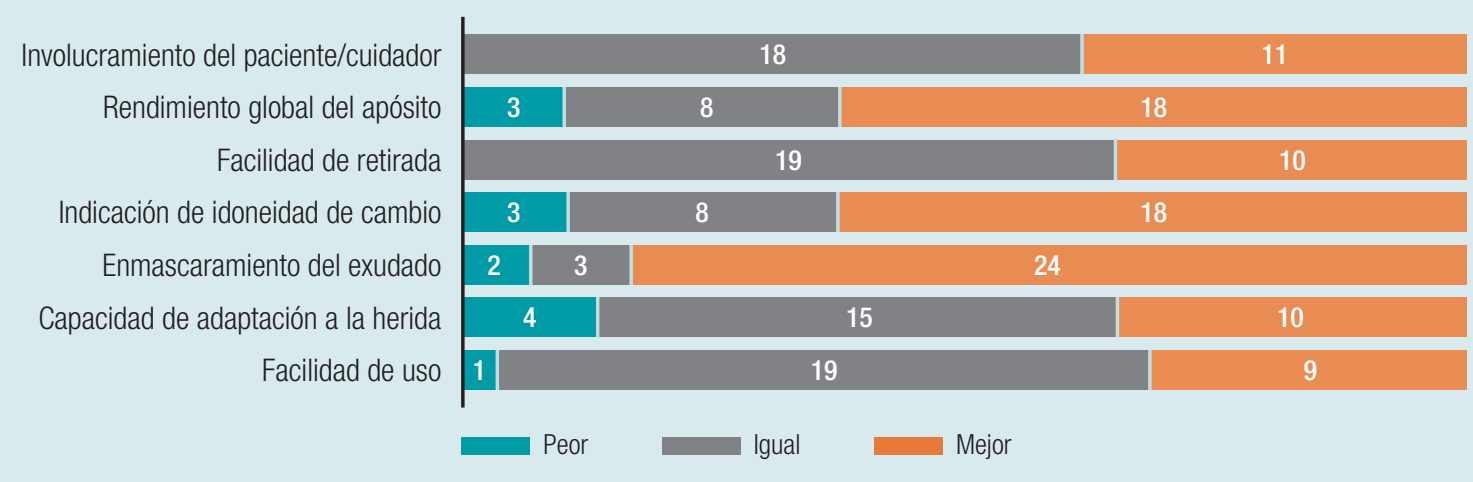

Figura 2. Valoración del apósito del estudio por el profesional de enfermería, en comparación con los apósitos previos (Galicia).

Tabla 3. Impacto del apósito ALLEVYN Life en la frecuencia de cambio, el área de la herida y el coste semanal por paciente (País Vasco)

\begin{tabular}{|l|c|c|c|c|}
\hline & Visita inicial (media \pm DE) & Visita final (media \pm DE) & Reducción & Valor de $\boldsymbol{p}$ \\
\hline Número de cambios de apósito & $2,1 \pm 0,6$ & $1,2 \pm 0,5$ & $43,5 \%$ & $<0,0001$ \\
\hline Área de la herida $\left(\mathrm{cm}^{2}\right)$ & $5,6 \pm 8,3$ & $3,5 \pm 7,6$ & $37,5 \%$ & 0,0179 \\
\hline Coste apósito semanal por paciente $(€)$ & $13,4 \pm 7,6$ & $8,6 \pm 5,9$ & $35,8 \%$ & 0,0079 \\
\hline Coste apósito-enfermería semanal por paciente $(€)$ & $63,1 \pm 19,3$ & $36,8 \pm 15,8$ & $41,7 \%$ & $<0,0001$ \\
\hline
\end{tabular}

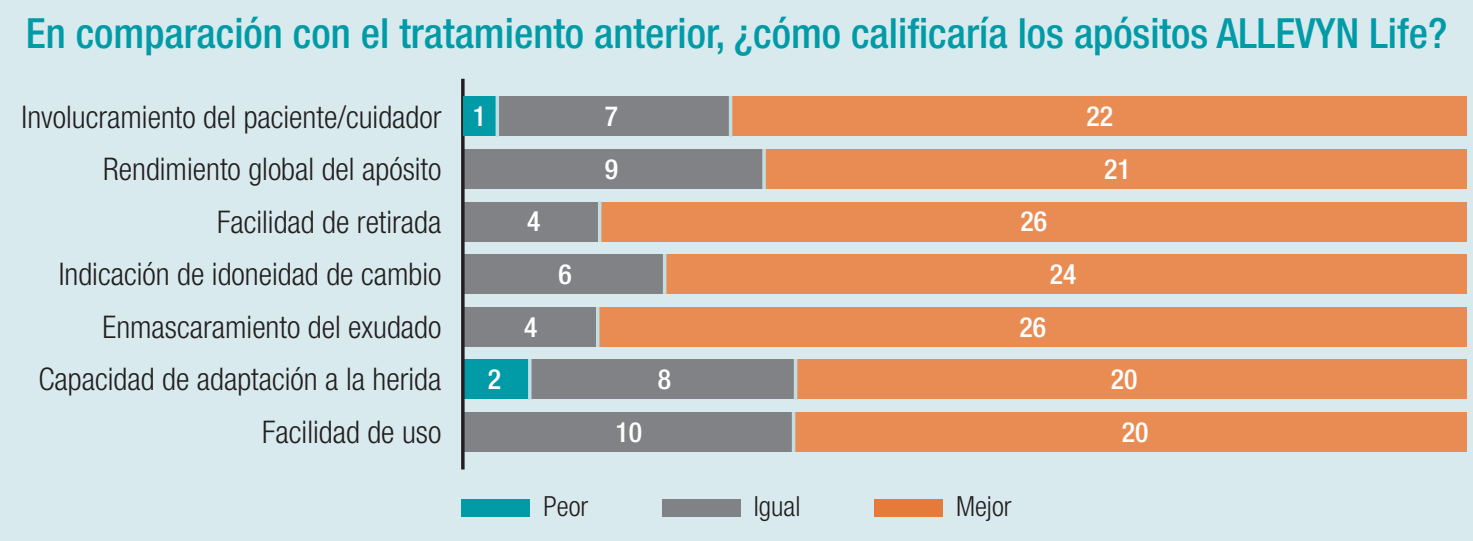

Figura 3. Valoración del apósito del estudio por el profesional de enfermería, en comparación con los apósitos previos (País Vasco).

Satisfacción del paciente. Los pacientes valoraron su experiencia global con el tratamiento previo de sus heridas como buena-muy buena en un 23,3\% de los casos. Tras el uso del apósito del estudio, la experiencia fue buena-muy buena en el $90 \%$ de los casos. En el $77,8 \%$ de los casos, el paciente indicó que sería "probable" o "muy probable" su recomendación del servicio a amigos o familiares, en caso de necesitarlo.

Opinión del profesional de enfermería. Según lo indicado por las enfermeras, ALLEVYN Life fue superior a los apósitos utilizados previamente en todas las características valoradas (fig. 3).

En el 93,3\% ( $\mathrm{n}=28)$ de los casos, la enfermera recomendaría el uso del apósito del estudio a su organización.

\section{IMPACTO EN LOS RESULTADOS CLÍNICOS}

La media del área de las heridas se redujo significativamente un 37,5\% a las 4 semanas (tabla 3).

\section{IMPACTO ECONÓMICO}

Como se muestra en la tabla 3, considerando únicamente el material utilizado en las curas, con ALLEVYN Life se obtuvo una reducción estadísticamente significativa del 35,8\% en el coste semanal por paciente. Del mismo modo, teniendo en cuenta el coste del tiempo de enfermería, se obtuvo una reducción estadísticamente significativa del coste semanal por paciente del $41,7 \%$. 
Tabla 4. Impacto del apósito ALLEVYN Life en la frecuencia de cambio, el área de la herida y el coste semanal por paciente (Extremadura)

\begin{tabular}{|l|c|c|c|c|}
\hline & Visita inicial (media \pm DE) & Visita final (media \pm DE) & Reducción & Valor de $p$ \\
\hline Número de cambios de apósito & $4,3 \pm 2,2$ & $1,8 \pm 1,3$ & $58,0 \%$ & $<0,0001$ \\
\hline Área de la herida $\left(\mathrm{cm}^{2}\right)$ & $23,2 \pm 37,2$ & $19,0 \pm 4,7$ & $18,1 \%$ & 0,0306 \\
\hline Coste apósito semanal por paciente (€) & $44,4 \pm 41,0$ & $17,4 \pm 23,6$ & $60,8 \%$ & 0,0176 \\
\hline Coste apósito-enfermería semanal por paciente (€) & $100,1 \pm 61,7$ & $40,8 \pm 39,4$ & $59,0 \%$ & 0,0012 \\
\hline
\end{tabular}

Tabla 5. Impacto del apósito ALLEVYN Life en la frecuencia de cambio, el área de la herida y el coste semanal por paciente (Comunidad Valenciana)

\begin{tabular}{|l|c|c|c|c|}
\hline & Visita inicial (media \pm DE) & Visita final (media \pm DE) & Reducción & Valor de $p$ \\
\hline Número de cambios de apósito & $3,8 \pm 1,9$ & $2,0 \pm 0,8$ & $48,7 \%$ & $<0,001$ \\
\hline Área de la herida $\left(\mathrm{cm}^{2}\right)$ & $9,1 \pm 6,9$ & $5,5 \pm 6,2$ & $39,6 \%$ & 0,0011 \\
\hline Coste apósito semanal por paciente $(€)$ & $24,1 \pm 31,6$ & $10,5 \pm 7,1$ & $56,3 \%$ & 0,0002 \\
\hline
\end{tabular}

\section{Estudio piloto en el Área de Salud de Mérida (Servicio Extremeño de Salud)}

\section{CARACTERÍSTICAS DE LAS HERIDAS}

Se incluyeron 20 pacientes con 25 heridas de diferente tipología (tabla 1), que en su mayoría $(84 \% ; \mathrm{n}=21)$ presentaban una evolución de 6 meses o menos.

IMPACTO EN LA PRÁCTICA CLÍNICA

El apósito del estudio se utilizó como apósito primario en el $60 \%$ $(\mathrm{n}=15)$ de las heridas, y fue utilizado como secundario en caso de necesidad de desbridamiento y de sospecha de infección. En estos casos, se utilizaron primariamente fibras gelificantes e hidrogeles.

Frecuencia de cambio de apósito. Con el uso del apósito del estudio, se obtuvo una reducción estadísticamente significativa en la frecuencia de cambio semanal del 58\% (tabla 4).

Satisfacción del paciente. Los pacientes valoraron su experiencia global respecto al tratamiento previo de sus heridas como buena-muy buena en un $18,5 \%$ de los casos, mientras que, tras su uso, fue buena-muy buena en el 69,6\%, Además, en el 86\% de los casos, el paciente indicó que sería "probable" o "muy probable" su recomendación del servicio a amigos o familiares en caso de necesitarlo.

Opinión del profesional de enfermería. Según la opinión de las enfermeras, ALLEVYN Life fue superior a los apósitos utilizados previamente en todas las características valoradas. En el 100\% ( $\mathrm{n}=25)$ de los casos, la enfermera recomendaría el uso del apósito del estudio a su organización.

\section{IMPACTO EN LOS RESULTADOS CLÍNICOS}

Desde la visita inicial, la media del área de las heridas se redujo significativamente un $18,1 \%$ a las 4 semanas (tabla 4 ).

\section{IMPACTO ECONÓMICO}

Considerando únicamente el material utilizado en las curas, con ALLEVYN Life se obtuvo una reducción estadísticamente significativa del $60,8 \%$ en el coste semanal por paciente (tabla 4). La reducción también fue estadísticamente significativa (59\%) al tener en cuenta el coste del tiempo de enfermería.

\section{Estudio piloto en la Comunidad Valenciana}

\section{CARACTERÍSTICAS DE LAS HERIDAS}

Se incluyeron 34 pacientes con 44 heridas de diferente tipología (tabla 1). El 47,4\% $(n=21)$ de las heridas presentaron una evolución superior a 6 meses.

\section{IMPACTO EN LA PRÁCTICA CLÍNICA}

El apósito del estudio se utilizó como apósito secundario en el 59,1\% $(\mathrm{n}=26)$ de las heridas, utilizándose primariamente en estos casos fibras gelificantes y antimicrobianos.

Frecuencia de cambio de apósito. Con el uso del apósito del estudio, se obtuvo una reducción significativa en la frecuencia de cambio semanal del 48,7\% (tabla 5).

Satisfacción del paciente. Los pacientes valoraron su experiencia global con el tratamiento previo de sus heridas como buena-muy buena en un $39,5 \%$ de los casos. Sin embargo, la experiencia con el apósito del estudio fue buena-muy buena en el 68,3\% de las heridas. En el 95,1\% de los casos, el paciente indicó que sería "probable" o "muy probable" su recomendación del servicio a amigos o familiares en caso de necesitarlo.

Opinión del profesional de enfermería. De acuerdo con la opinión de las enfermeras, ALLEVYN Life fue superior a los utilizados previamente en todas las características valoradas, a excepción de la "capacidad del apósito en adaptarse al sitio de la herida”, considerándola igual (en el $59,1 \%$ de las heridas) o mejor (40,9\%) que los apósitos previos.

En el 95,5\% ( $\mathrm{n}=42)$ de los casos, la enfermera recomendaría el uso del apósito del estudio a su organización.

\section{IMPACTO EN LOS RESULTADOS CLÍNICOS}

Con el apósito del estudio, se obtuvo una reducción significativa del área de la herida del 39,6\% a las 4 semanas (tabla 5).

\section{IMPACTO ECONÓMICO}

La tabla 5 muestra que, considerando el material utilizado en las curas, con ALLEVYN Life se obtuvo una reducción estadísticamente significativa del $56,3 \%$ en el coste semanal por paciente, en comparación con los apósitos previos. 


\section{- DISCUSIÓN}

El presente artículo muestra el impacto sobre la eficiencia en la cura de heridas que tiene el uso de un apósito innovador en la práctica clínica real. El cambio al apósito de estudio, tras los tratamientos utilizados previamente, condujo a una reducción del número de cambios de apósito semanales. Esta reducción de la frecuencia tuvo un impacto tanto en el número de curas realizadas por el profesional de enfermería como en los materiales utilizados en las mismas, por lo que se consiguió espaciar las visitas y reducir el coste asociado a los materiales y al tiempo de enfermería. La experiencia global del paciente mejoró con el cambio de apósito, mientras que, desde la perspectiva del profesional de enfermería, la capacidad de indicación de cambio y de enmascaramiento del exudado de ALLEVYN Life fueron características diferenciales frente a los tratamiento previos. Adicionalmente, se obtuvo una disminución significativa del área de la herida a las 4 semanas, lo que sugiere que el tratamiento es efectivo a corto plazo.

En el estudio se incluyeron pacientes de centros de salud y sociosanitarios, que presentaban mayoritariamente úlceras en EE. II. y LPP, lo que refleja la prevalencia en la vida real de este tipo de heridas crónicas y su impacto asistencial. La proporción de úlceras en EE. II. fue mayor en Galicia y País Vasco. Sin embargo, en la Comunidad Valenciana hubo mayor proporción de LPP, mientras que en Extremadura no hubo diferencias.

Estudios previos llevados a cabo en AP en otras zonas de España muestran resultados diversos. En un estudio realizado en Murcia, la proporción de LPP atendidas (44\%) fue superior a la proporción de úlceras vasculares $(29 \%)^{26}$. En otro estudio llevado a cabo en Castellón, se atendió a pacientes con mayor proporción de úlceras en EE. II. (29\%) que de LPP $(14,3 \%)^{27}$. A pesar de las diferencias observadas en nuestro estudio entre regiones, los resultados globales indican una proporción similar de úlceras en EE. II. (39,8\%) y LPP (31,3\%).

Con el uso de ALLEVYN Life, se redujo significativamente la frecuencia de cambio en todas las regiones. El número medio de cambios semanales previos a su uso en Galicia y País Vasco fue similar al encontrado en un estudio llevado a cabo en Reino Unido, en el que la frecuencia fue de 2,4 cambios por semana ${ }^{28}$. Otros estudios, como el realizado por Jorgensen et al. ${ }^{29}$, en pacientes ambulatorios con tratamiento de úlceras de diferente tipología, seńalaban una media de 3,5 cambios de apósito semanales, o el estudio de Gottrup et al. ${ }^{30}$, con 2,5-3,1 cambios. De las cuatro regiones estudiadas, Extremadura fue la que obtuvo una mayor frecuencia de cambio de apósito previa, y en la que más se redujo esta con ALLEVYN Life (58\%), seguida de Comunidad Valenciana $(48,7 \%)$, obteniendo una media de 1,2-2 cambios semanales en todas las regiones.

Un estudio piloto realizado en Reino Unido en pacientes con heridas crónicas, en el que se utilizó este apósito, obtuvo resultados similares, reduciendo la frecuencia desde 4,3 cambios semanales de media previos hasta 2,2 cambios $^{19}$.

Desde el punto de vista económico, al considerar únicamente el material utilizado en las curas, se obtuvo una reducción significativa del coste semanal por paciente en País Vasco, Extremadura y Comunidad Valenciana, pero no así en Galicia, donde se observó una tendencia a la reducción del coste sin diferencias estadísticamente significativas. No obstante, al considerar el coste del tiempo de enfermería para curas ambulatorias, en esta región si se obtuvo una reducción del coste semanal por paciente significativa, cercana al 30\%. Del mismo modo, en las otras dos regiones en las que se tuvo en cuenta el coste del tiempo de enfermería para curas ambulatorias, País Vasco y Extremadura, la disminución del coste fue superior al $40 \%$, siendo estadísticamente significativa en ambos casos.

Estos resultados reafirman la importancia del impacto económico que supone disminuir la frecuencia de cambio de apósito en la práctica clínica, como la evidencia previa indica ${ }^{1}$. A nivel de gestión sanitaria, esto podría suponer, no solo reducir el coste asociado a las curas, sino también una minimización del coste de oportunidad, que, en salud, hace referencia al beneficio al que se renuncia asignando un recurso sanitario a una actividad determinada ${ }^{1}$. Aquellos recursos que se utilizan en exceso con una diferencia clínica muy pequeña, existiendo una alternativa que consiga evitarlo, suponen un alto coste de oportunidades, por tanto menor beneficio para los pacientes y mayor ineficiencia del sistema ${ }^{31}$. Por ello, esto implica la posibilidad de que los recursos utilizados hasta el momento en la práctica clínica habitual, ante una mayor frecuencia de cambio de apósito, podrían lograr más valor en otras actividades del cuidado de las heridas o en otras actividades propias de las enfermeras, si se redujera dicha frecuencia.

Por otra parte, se analizó la reducción en el área de las heridas como resultado clínico, y se obtuvo una reducción significativa con el apósito del estudio. El porcentaje de reducción se considera un indicador de cicatrización relevante en la monitorización de las heridas ${ }^{32}$. Estudios previos llevados a cabo en pacientes con úlceras venosas indican que porcentajes de reducción iguales o superiores a un 30\% tras 4 semanas constituyen un buen predictor de cicatrización ${ }^{32}$. A las 4 semanas, nuestros resultados fueron superiores a ese porcentaje en la mayoría de las regiones.

Los pacientes atendidos valoraron su experiencia global del cuidado de las heridas como buena o muy buena tras el uso de ALLEVYN Life en la mayoría de los casos. La valoración más positiva la encontramos en País Vasco, donde los pacientes valoraron su experiencia como buena o muy buena en el $90 \%$ de los casos, con un aumento de la satisfacción de cerca del $67 \%$ en comparación con los apósitos previos. La consideración de las preferencias del paciente se ha convertido en un componente clave en el rediseńo de los procesos de salud para mejorar los resultados y la seguridad. La evidencia previa seńala la importancia de alinear las prioridades del paciente con la investigación de nuevos productos y tratamientos para obtener mejores resultados en el cuidado de las heridas ${ }^{33}$.

En todas las regiones, el profesional de enfermería consideró los apósitos ALLEVYN Life superiores al tratamiento anterior en cuanto a rendimiento global del apósito, indicación de idoneidad de cambio y enmascaramiento del exudado, e indicaron que recomendarían su uso dentro de su organización con un alto porcentaje (80-100\%). El profesional de enfermería debe disponer de productos innovadores para la cura de heridas que les permita prolongar los intervalos entre visitas y faciliten el empoderamiento del paciente en su autocuidado ${ }^{14}$. Además, estos apósitos cumplirían con las características del apósito ideal, que incluye, entre otras, la incorporación de sensores o alertas del rendimiento del apósito, la necesidad de cambio y el estado de la herida ${ }^{13}$.

El estudio no está exento de limitaciones. En primer lugar, el tamaño de la muestra fue estimado para realizar estudios piloto en cada región. Aunque el análisis ha permitido identificar cambios estadísticamente significativos en la frecuencia de cambio, no se han podido realizar comparaciones por tipología de heridas. No obstante, un análisis conjunto de todas las heridas podría aportar información adicional sobre cambios acontecidos en las más prevalentes o aquellas que requieren más recursos.

En segundo lugar, el estudio no disponía de grupo control con el que comparar durante el seguimiento, y las heridas incluidas presentaban diversa tipología y diferentes periodos de evolución, lo que podría haber influido en los resultados obtenidos. Sin embargo, todas las heridas 
recibieron un tratamiento previo al apósito del estudio, que podría considerarse el control. Además, la heterogeneidad de las heridas es representativa de la realidad de la práctica clínica, por lo que los resultados obtenidos aportan una evidencia relevante a nivel clínico y de gestión de los recursos sanitarios.

En tercer lugar, no se recogieron datos de eficacia de cicatrización que complementaran los resultados del estudio. Sin embargo, aunque evaluar la eficacia no era un objetivo del estudio, sí se obtuvo un alto porcentaje de reducción del área de las heridas a las 4 semanas, lo que constituye un buen predictor de cicatrización.

Por último, debido al pequeño número de pacientes incluido en el estudio en residencias sociosanitarias, no se han podido comparar resultados en diferentes entornos, y las heridas atendidas en estos centros han sido incluidas en el análisis y tratadas del mismo modo que las heridas atendidas en los centros de salud participantes.

\section{- CONCLUSIÓN}

Este estudio muestra que el apósito con indicador de cambio y capa enmascaradora, ALLEVYN Life, en el tratamiento de heridas en práctica clínica real, disminuye significativamente el número de cambios de apósito semanales, lo que permite prolongar los intervalos entre visitas y reducir los costes asociados. Al mismo tiempo, se obtiene una reducción significativa del área de la herida a las 4 semanas, lo que sugiere una tendencia hacia la cicatrización. Con el apósito de estudio mejora la satisfacción del paciente con el manejo de sus heridas, así como la opinión del profesional de enfermería, en comparación con los apósitos utilizados anteriormente. Esto se traduce en una mayor eficiencia en el proceso asistencial de las curas de heridas y en una mejora de la calidad de la atención.

\section{Conflicto de intereses}

Tim Styche, Francisco José Valenzuela y Leticia Muñoz García declaran que son empleados de Smith+Nephew. El resto de los autores son investigadores independientes y no tienen relaciones financieras relacionadas con este artículo.

\section{Agradecimientos}

Los autores agradecen a Outcomes'10 su apoyo como consultoría científica en el análisis de datos y la edición de este manuscrito. Los autores también desean agradecer la colaboración de los siguientes profesionales de la salud en la recopilación de los datos para el estudio: de Bilbao, a Irene Sainz Rozas Aparicio, Elena Andreu Muńoz, Julia Huerta Fernández, Miriam Díaz de Espada López de Ondategui, Peter Tadorian Ramos; de la Comunidad Valenciana, a Verónica Lozano Rodríguez, María Ángeles Pérez Corcoles, Vicente Martínez López, José Vicente Cardona Gómez, Concha Josa Conejos, Leticia García Laporta, Estrella Villar Vera, Lucía Fernández Bielsa, Inma Portolés Rincón, Nieves Puig Zanon y Celia Rodado Guirado; de Extremadura, a Zulaica Gutiérrez Méndez, María Jesús Sánchez Sánchez, Ana María García Soleto, María del Carmen García Casado, Francisco Domínguez Arévalo, José Antonio Moyano Muñoz-Reja, Lorena Hornero Zabala y Severiano García Sánchez; de Galicia, a Yolanda Rizzo Losada, Íria Vázquez Cabido, Francisco Javier Barreiros Ribao, María Cid Gómez, Ángel Rodríguez Álvarez, María Floren Vale Iglesias, Josefa Gregoria Paz Lorenzo, Isabel González Lameiro, Soledad Valencia

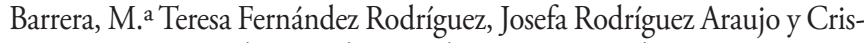
tina Suárez Fernández. Finalmente, los autores agradecen a Juan Cano Cánovas el diseño y desarrollo del estudio, y a M. Gloria Lorente Granados y a +QCuidar como empresa de servicios sanitarios, por la formación y seguimiento clínico en las zonas de Orense y Mérida.

\section{BIBLIOGRAFÍA}

1. Lindholm C, Searle R. Wound management for the 21 st century combining effectiveness and efficiency. Int Wound J. 2016;13:5-15

2. International Wound Infection Institute (IWIII). Wound Infection in Clinical Practice. Wounds International. 2016

3. García-Fernández F, Enric Torra i Bou J, Soldevilla Agreda JJ, Pancorbo-Hidalgo P. Prevalencia de lesiones por presión y otras lesiones cutáneas relacionadas con la dependencia en centros de atención primaria de salud de España en 2017. Gerokomos. 2019;30(3):134-41.

4. Torra i Bou JE, Soldevilla Agreda JJ, Rueda López J, Verdú Soriano J, Roche Rebollo E, Arboix i Perejamo M, et al. Primer Estudio Nacional de Prevalencia de Úlceras de Pierna en España. Estudio GNEAUPP-UICF-Smith \& Nephew 2002-2003. Gerokomos 2004;15(4):230-47.

5. Marinel.Io Roura J, Verdú Soriano J, coord. Conferencia nacional de consenso sobre las úlceras de la extremidad inferior (C.O.N.U.E.I.) Documento de consenso 2018. $2^{\text {a }}$ ed. Madrid: Ergon; 2018.

6. Torra-Bou J, García-Fernández F, Pérez-Acevedo G, Sarabia-Lavin R Paras-Bravo P, Soldevilla-Ágreda J, et al. El impacto económico de las lesiones por presión. Revisión bibliográfica integrativa. Gerokomos. 2017:28(2):83-97.

7. Kronert GT, Roth H, Searle RJ. The impact of introducing a new foam dressing in community practice. EWMA J. 2016;16(2):7-12

8. Blackburn J, Stephenson J, Atkin L, Southern TOM, Ousey K. Exploring and understanding challenges in clinical practice: appropriate dressing wear time. Wounds UK. 2018;14(5):56-64.

9. Seckam A. A multicentre, observational evaluation of the product characteristics of two absorbent foam dressings. Br J Nurs. 2019:28(12):12-7.

10. Benbow M. The expense of exudate management. Br J Nurs 2015;24(15):S8.

11. Upton D, Solowiej K, Hender C, Woodyatt KY. Stress and pain associated with dressing change in patients with chronic wounds. J Wound Care. 2012;21(2):53-61.
12. Woo K, Santos VLC de G, Alam T. Optimising quality of life for people with non-healing wounds. Wounds Int. 2018;9(3):6-14.

13. World Union of Wound Healing Societies (WUWHS). Consensus document. Wound exudate: effective assessment and management. Wounds International. 2019.

14. Wang R, Peng Y, Jiang Y, Gu J. Managing chronic wounds during novel coronavirus pneumonia outbreak. Burn Trauma. 2020;8:4-7.

15. Özker E, Erkin A, Aslan HM, Kurtoğlu T, Çayır MÇ, Akay T, et al. Wound treatment strategies during COVID-19 pandemic: An expert opinion. Turkish J Vasc Surg. 2020;29.

16. European Wound Management Association (EWMA) website COVID-19 related information and news [Internet]. 2020 [citado 25 de junio de 2020]. Disponible en: https://ewma.org/covid-19/

17. Chamorro AM, Vidal Thomas MC, Mieras AS, Leiva A, Martínez MP Hernández Yeste MMS. Multicenter randomized controlled trial comparing the effectiveness and safety of hydrocellular and hydrocolloid dressings for treatment of category $\|$ pressure ulcers in patients at primar and long-term care institutions. Int J Nurs Stud. 2019;94:179-85.

18. Forni C, D'Alessandro F, Gallerani P, Genco R, Bolzon A, Bombino $C$, et al. Effectiveness of using a new polyurethane foam multi-layer dressing in the sacral area to prevent the onset of pressure ulcer in the elderly with hip fractures: A pragmatic randomised controlled trial. Int Wound J. 2018:15(3):383-90.

19. Joy H, Bielby A, Searle R. A collaborative project to enhance efficiency through dressing change practice. J Wound Care 2015:24(7):312-7.

20. Viechtbauer W, Smits L, Kotz D, Budé L, Spigt M, Serroyen J, et al A simple formula for the calculation of sample size in pilot studies. J Clin Epidemiol. 2015;68(11):1375-9.

21. Lancaster GA, Dodd S, Williamson PR. Design and analysis of pilot studies: Recommendations for good practice. J Eval Clin Pract. 2004;10(2):307-12
22. Diario Oficial de Galicia. DECRETO 56/2014, de 30 de abril, por e que se establecen las tarifas de los servicios sanitarios prestados en los centros dependientes del Servicio Gallego de Salud y en las fundaciones públicas sanitarias. [Internet]. 2014 [citado 26 de junio de 2020]. Disponible en: https://www.xunta.gal/dog/Publicados/2014/20140521/AnuncioC3K1-140514-0001_es.html

23. Instituto Nacional de Estadística. Actualización de rentas con el IPC general (sistema IPC base 2016) para periodos anuales completos [Internet]. [citado 26 de junio de 2020]. Disponible en: http://www. ine.es/calcula/calcula.do

24. Boletín Oficial del País Vasco. Acuerdo de 15 de febrero de 2017, del Consejo de Administración del Ente Público Osakidetza - Servicio Vasco de salud, por el que se aprueban las tarifas por prestación de servicios sanitarios y docentes a terceros obligados al pago durante el ejercicio 2 [Internet]. 2017 [citado 26 de junio de 2020]. Disponible en: https://www.euskadi.eus/gobierno-vasco/-/acuerdo/ acuerdo-de-15-de-febrero-de-2017-del-consejo-de-administraciondel-ente-publico-osakidetza-servicio-vasco-de-salud-por-el-que-seaprueban-las-tarifas-por-prestacion-de-servicios-sanitarios-y-docentes-a-terceros-obligados-al-pago-durante-el-ejercicio-201/

25. Diario Oficial de Extremadura. Ley $1 / 2018$, de 23 de enero, de Presupuestos Generales de la Comunidad Autónoma de Extremadura para 2018. [Internet]. 2018 [citado 26 de junio de 2020]. Disponible en: http:// portaltributario.juntaex.es/PortalTributario/c/document_library/get_file?uuid=7b330e80-66c6-4a06-9ac5-a93a41bb1d23\&groupld=10136

26. Avilés Aranda J, Moreno Pina J, Palomar Llatas F, Peñalver Hernández F, Vivancos Oliva V, Pastor Macia J. Heridas crónicas en un área de salud de murcia. Enferm Dermatol. 2016;10(27):19-24.

27. Guinot-Bachero J, Herrera-Herzog E, Ibáñez-Valero I, Sorribes-Lengua M, Adsuara-Molina J, Garralón-Pérez A, et al. Heridas en consultas de enfermería: ¿qué lesión genera más carga de trabajo? Estudio observacional urbano. Gerokomos. 2017;28(1):42-8. 
Verónica Tiscar González, María José Menor Rodríguez, Carlos Rabadán Sainz, Mercedes Fraile Bravo, Grupo Life, Tim Styche, Francisco José Valenzuela Ocaña y Leticia Muñoz García Eficiencia de un apósito innovador en la cura de heridas: reducción de la frecuencia de cambio y del coste semanal por paciente

28. Vowden K, Vowden P, Posnett J. The resource costs of wound care in Bradford and Airedale primary care trust in the UK. J Wound Care. 2009;18(3):93-102.

29. Jorgensen S, Nygaard R, Posnett J. Meeting the challenges of wound care in Danish home care. J Wound Care. 2013;22(10):540-5
30. Gottrup F, Henneberg E, Trangbæk R, Bækmark N, Zøllner K, Sørensen J. Point prevalence of wounds and cost impact in the acute and community setting in Denmark. J Wound Care. 2014:22(8):413-22.

31. Gray TA, Rhodes S, Atkinson RA, Rothwell K, Wilson P, Dumville $\mathrm{JC}$, et al. Opportunities for better value wound care: A multiservice, cross-sectional survey of complex wounds and their care in a UK community population. BMJ Open. 2018:8(3):1-9.

32. Flanagan M. Wound measurement: can it help us to monitor progression to healing? J Wound Care. 2003:12(5):189-94.

33. Corbett LQ, Ennis WJ. What Do Patients Want? Patient Preference in Wound Care. Adv Wound Care. 2014;3(8):537-43. 